BNL-114245-2017-JA

\title{
On determination of charge transfer efficiency of thick, fully depleted CCDs with 55Fe x-rays
}

\author{
D. Yates, A. Nomerotski
}

Submitted to Journal of Instrumentation

August 2017

Physics Department

\section{Brookhaven National Laboratory}

\author{
U.S. Department of Energy \\ USDOE Office of Science (SC), \\ High Energy Physics (HEP) (SC-25)
}

Notice: This manuscript has been authored by employees of Brookhaven Science Associates, LLC under Contract No. DE-SC0012704 with the U.S. Department of Energy. The publisher by accepting the manuscript for publication acknowledges that the United States Government retains a non-exclusive, paid-up, irrevocable, world-wide license to publish or reproduce the published form of this manuscript, or allow others to do so, for United States Government purposes. 


\section{DISCLAIMER}

This report was prepared as an account of work sponsored by an agency of the United States Government. Neither the United States Government nor any agency thereof, nor any of their employees, nor any of their contractors, subcontractors, or their employees, makes any warranty, express or implied, or assumes any legal liability or responsibility for the accuracy, completeness, or any third party's use or the results of such use of any information, apparatus, product, or process disclosed, or represents that its use would not infringe privately owned rights. Reference herein to any specific commercial product, process, or service by trade name, trademark, manufacturer, or otherwise, does not necessarily constitute or imply its endorsement, recommendation, or favoring by the United States Government or any agency thereof or its contractors or subcontractors. The views and opinions of authors expressed herein do not necessarily state or reflect those of the United States Government or any agency thereof. 


\section{On determination of charge transfer efficiency of thick, fully depleted CCDs with ${ }^{55} \mathrm{Fe}$ x-rays}

\section{Yates, ${ }^{a, b, 1}$ I. Kotov ${ }^{a}$ and A. Nomerotski ${ }^{a}$}

${ }^{a}$ Brookhaven National Laboratory, Upton, NY, 11973, U.S.A.

${ }^{b}$ Pacific University,

Forest Grove, OR, 97116, U.S.A.

E-mail: yate3961@pacificu.edu

AвstRACT: Charge transfer efficiency (CTE) is one of the most important CCD characteristics. This paper examines ways to optimize the algorithms used to analyze ${ }^{55} \mathrm{Fe}$-ray data on the CCDs, as well as explores new types of observables for CTE determination that can be used for testing LSST CCDs. The observables are modeled employing simple Monte Carlo simulations to determine how the charge diffusion in thick, fully depleted silicon affects the measurement. The data is compared to the simulations for one of the observables, integral flux of the x-ray hit.

KEYwords: Charge transport and multiplication in solid media; Detector modelling and simulations II (electric fields, charge transport, multiplication and induction, pulse formation, electron emission, etc); Photon detectors for UV, visible and IR photons (solid-state) (PIN diodes, APDs, Si-PMTs, G-APDs, CCDs, EBCCDs, EMCCDs etc); Image processing 


\section{Contents}

1 Introduction 1

2 Finding of x-ray hits 2

3 Simulation of charge transfer efficiency 4

4 CTE measurement in the ${ }^{55} \mathrm{Fe}$ data $\quad 7$

5 Conclusions 9

\section{Introduction}

The digital sensor array for the LSST camera will consist of 21 science rafts of nine CCDs (ChargeCoupled Devices) each, totaling 189 CCD sensors on the focal plane. Each CCD consists of approximately 16 million $10 \times 10 \mu \mathrm{m}^{2}$ pixels, giving a three gigapixel image [1]. CCDs are divided into two halves of eight sections each to satisfy a stringent specification of two second readout time for the full focal plane and low noise of 8 electrons for a 15 second exposure time at $-100^{\circ} \mathrm{C}$. High quantum efficiency is required for light in $320-1080 \mathrm{~nm}$ range, achieved by using fully depleted, $100 \mu \mathrm{m}$ thick CCDs and thin entrance window [2].

The charge transfer efficiency (CTE) of a CCD is one of the most important characteristic that must be known and optimized to ensure CCD sensors give accurate representation of astronomical data. CTE is defined as the ratio of electrons transferred between two neighboring pixels to total electrons contained in the pixel before the transfer. CCDs perform both serial and parallel transfers during charge readout, each with its own CTE [3]. Sensors for the LSST camera require high CTE, 0.999995 for 500 serial transfers and 0.999997 for 2000 parallel transfers, to ensure accurate readout while imaging [4]. This study is focused mostly on the parallel charge transfer.

Soft $\mathrm{x}$-rays from ${ }^{55} \mathrm{Fe}$ are the commonplace method used to determine properties of CCDs, including CTE. X-rays from ${ }^{55} \mathrm{Fe}$ deposit around $1620 \mathrm{e}^{-}$and $1778 \mathrm{e}^{-}$in the silicon sensor, corresponding to the $5.9 \mathrm{keV} \mathrm{K}_{\alpha}$ and $6.5 \mathrm{keV} \mathrm{K}_{\beta}$ lines, respectively [3]. The probability for the $\mathrm{x}$-rays to interact in 100 micron of silicon is about $97 \%$. Due to the drift of photoelectrons in $100 \mu \mathrm{m}$ thick silicon, there is approximately $3.6 \mu \mathrm{m}$ (sigma) of charge diffusion, resulting in charge spread into adjacent pixels. This makes the determination of CTE less straightforward as the signal can appear in the trailing pixels not only due to the charge transfer inefficiency, but also due to the diffusion.

The most common CTE determination method uses x-rays with known charge deposition in CCD (like for ${ }^{55} \mathrm{Fe}$ ) and analyzes total hit flux as a function of number of pixel transfers in order to determine global CTE. Another popular method of CTE determination, know as extended pixel edge response (EPER), examines deferred charge in the extended pixel region of the CCD (so called overscans) to determine CTE [3]. This study, described here, is in search of new measurement 
algorithms that can be made on ${ }^{55} \mathrm{Fe}$ data to determine global CTE of the CCD sensor taking into account additional smearing due to the diffusion. These studies include both optimization of the ${ }^{55} \mathrm{Fe}$ hit finding and also evaluation of observables that can be constructed using the pixel by pixel information in the found hits.

\section{Finding of x-ray hits}

Initial analysis looks to optimize the x-ray hit finding algorithm used to determine locations and other properties of $\mathrm{x}$-ray hits in the CCD. The data sample used for these studies was collected with a LSST CCD-250 prototype sensor, e $2 \mathrm{v}-113-03$, produced by e2v [5]. The sensor, at $-120^{\circ} \mathrm{C}$ inside a cryostat and biased at $-70 \mathrm{~V}$, was read out with Reflex controller using RTS-2 software [6] in a setup described in details elsewhere [7].

The algorithm searches for a set number of adjacent pixels above the threshold and identifies them as a hit region. The program then creates a stamp around the hit, with the number of pixels on each side of the hit being the specified growth parameter. Examples of x-ray hits in the CCD with growth factor of two applied are shown in figure 1. About 3500 hits were recorded in the CCD in each exposure. 2-D Gaussian fitting then occurs on the hit using ngmix software [8] in order to establish flux and other parameters for each hit.

Prior to identifying $\mathrm{x}$-ray hits, the algorithm determines the CCD noise defined as clipped standard deviation (stdevclip) of pixel flux in images taken with no exposure time (bias images). The hit finding algorithm relies on three main parameters to determine x-ray hits: pixel threshold, minimum number of adjacent pixels above the threshold and growth factor. To determine optimal values for each of these three parameters, a scan is conducted over the corresponding parameters space, selecting several values for the growth and number of adjacent pixels parameters and plotting the number of reconstructed x-ray hits as function of the threshold expressed in multiples of the CCD noise.

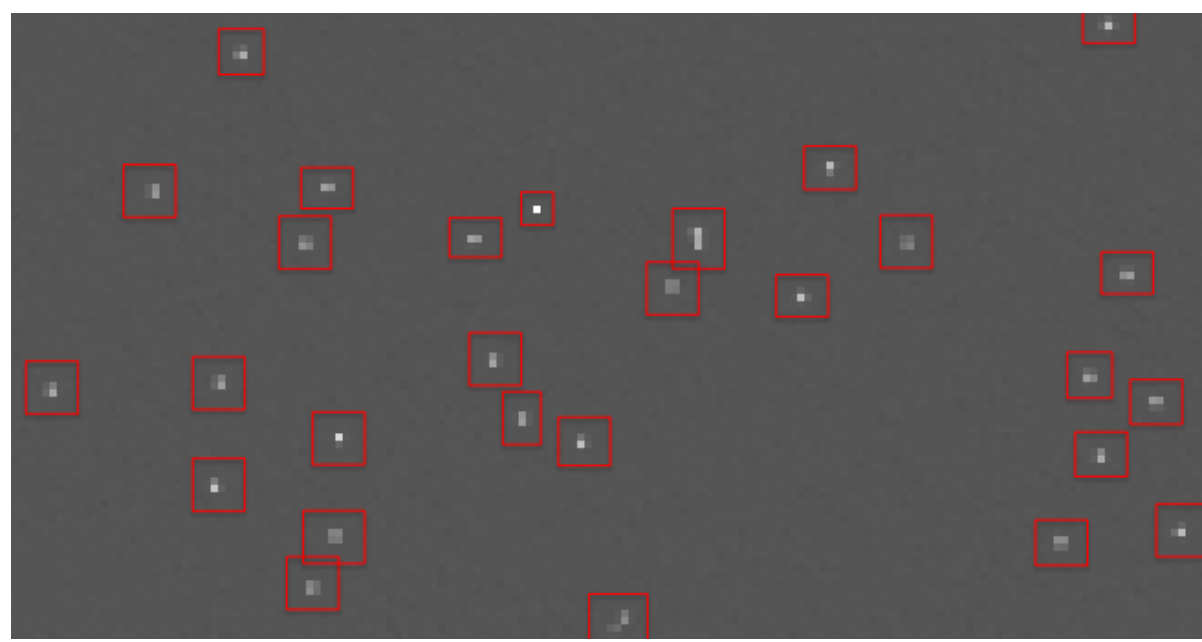

Figure 1. Examples of found $x$-ray hits in CCD with associated stamps, or footprints, which include pixels used in the following data analysis.

Figure 2 shows the number of identified $x$-ray hits as function of the threshold for varied growth factor at minimum number of adjacent pixels equal to two. The amount of found $\mathrm{x}$-ray hits decreases 
with both increased threshold and growth factor, as expected. Increased threshold removes more pixels from consideration as overlapping stamps are merged by the algorithm to a single stamp. Increased growth factor leads to merging of multiple $\mathrm{x}$-ray hits into a single hit region. Both effects lower the total number of identified hits in the image.

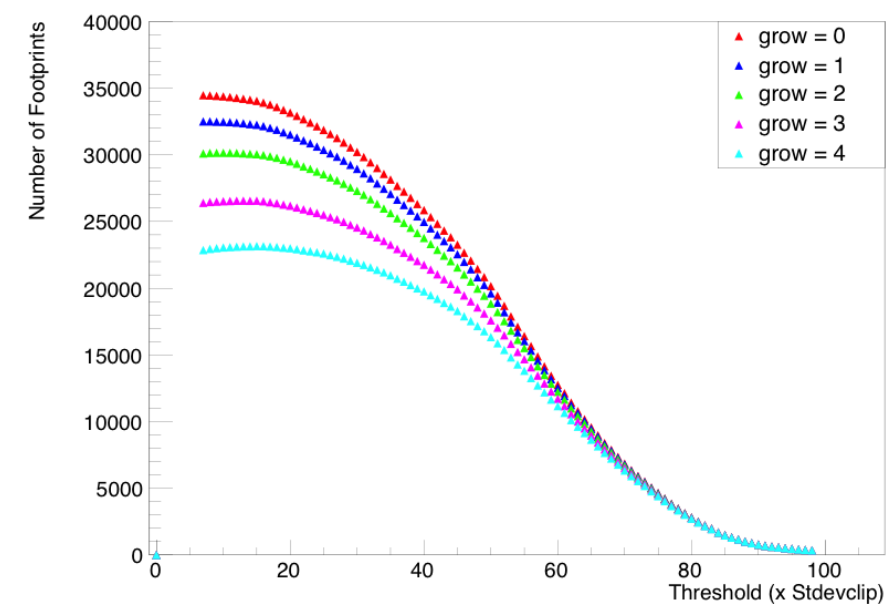

Figure 2. Number of identified $x$-ray hits as function of the threshold for varied growth factor (grow) at minimum number of adjacent pixels over the threshold equal to two.

Figure 3 shows the number of identified $\mathrm{x}$-ray hits as function of the threshold for varied number of minimum adjacent pixels (npixMin) at grow factor equal to two. Similarly to the previous figure, a decline in identified hits with an increase in the threshold and minimum pixel number is expected.

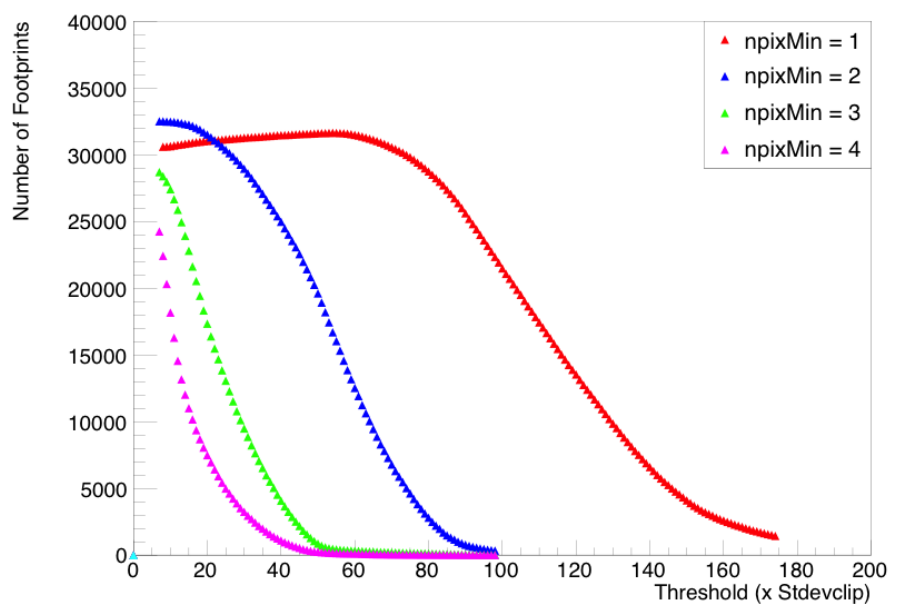

Figure 3. Number of identified $x$-ray hits as function of the threshold for varied minimum adjacent pixels (npixMin) at growth factor equal to two.

When identifying x-ray hits with only one pixel over threshold, there exists a plateau where the number of $\mathrm{x}$-ray hits does not depend strongly on the threshold, meaning that almost all the $\mathrm{x}$-ray hits in the exposure are found. When higher minimum number of adjacent pixels are required, the x-ray hits, which occur near the pixel center, could escape identification by the algorithm at higher 
threshold levels, as seen in figure 3, due to low signal in adjacent pixels as about $70 \%$ of the charge in this case is deposited in the central pixel [9]. By requiring only a single pixel triggered above a given threshold, the threshold trigger value becomes less critical in the data analysis.

\section{Simulation of charge transfer efficiency}

To examine and compare several algorithms for CTE determination, simple Monte Carlo simulations were implemented. The starting point was to create a virtual $x$-ray randomly positioned within a pixel in the CCD with flux normally distributed around its central value of 1620 electrons with 20 electrons standard deviation. The shape of the deposited charge was modeled with 2-D Gaussian with sigma equal to 3.6 micron, in agreement with diffusion of ionization in 100 microns of silicon. The signal then was distributed among the pixels according to the above model. The hit shape has a strong dependence on the position of the x-ray hit within the central pixel [9].

To model CTE the simulation then transfers the hit between the pixels with a predefined CTE value by adjusting the charges in corresponding pixels. It also tracks characteristics of each hit over 2000 transfers. Virtual hits are fit with 2-D Gaussian using ngmix software [8] every 100 transfers, and hit characteristics such as hit flux, ellipticity and centroid coordinates are recorded for further evaluation. The individual pixel fluxes are also recorded based on the raw pixel information without relying on the fit. The number of simulated x-ray hits was chosen to be equal to 100,000 to keep statistical uncertainties low enough.

This analysis looks at five specific observables relevant for the CTE determination: central pixel flux, ellipticity of hit, integral of flux over the $3 \times 3$ pixel region, integral of flux using $2 \mathrm{D}$ Gaussian fit, and flux difference between trailing and leading pixel, all schematically explained in figure 4. The analysis evaluates how the observables behave at different CTE.
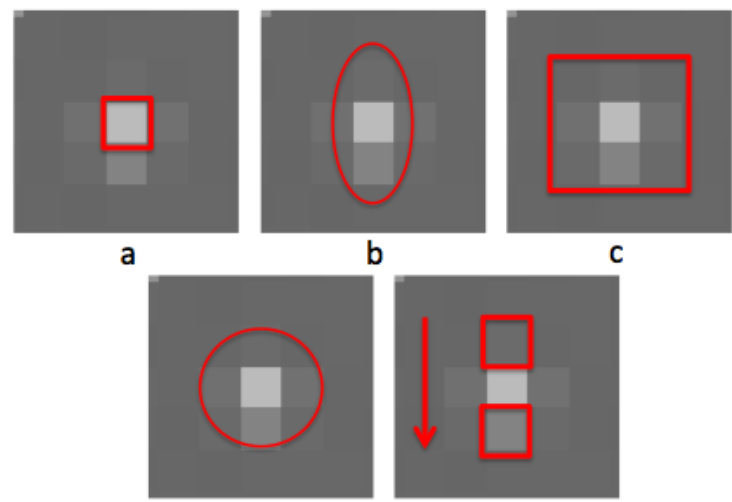

d

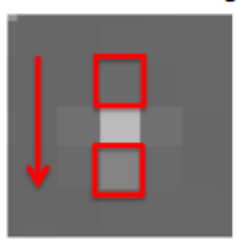

Figure 4. Five possible measurements of CTE: a) central pixel flux, b) hit ellipticity, c) integral of flux over the $3 \times 3$ pixel region, d) 2-D Gaussian flux fit, e) flux difference between trailing and leading pixel (arrow indicates transfer direction).

Figure 5 shows a plot of the difference in flux between trailing and leading pixel (dFlux) versus the number of transfers for several CTE values. As expected, the flux difference is increasing with increased number of transfers. The dependence is almost linear, especially for the highest CTE 
values. Fitting the series with a linear function and plotting the function slope in figure 5 against the corresponding charge transfer inefficiency (CTI $=1-\mathrm{CTE})$, one can evaluate the dependence of this observable, the flux difference, on CTI as shown in figure 6. Good correlation exists between the slope and CTI, with a linear relationship well describing the points. This suggests that the differential flux is a good CTE estimator for the ${ }^{55} \mathrm{Fe} x$-ray data.

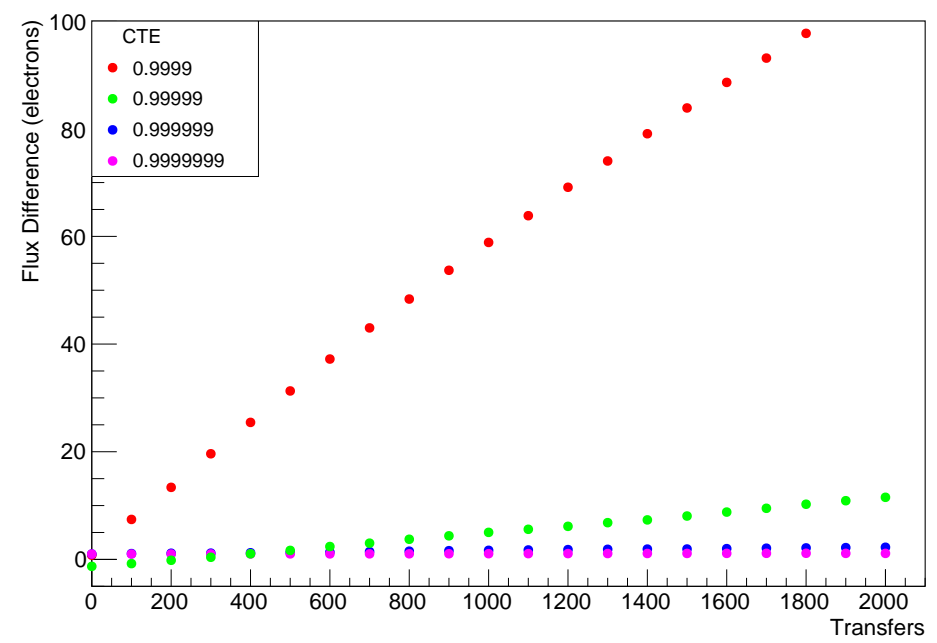

Figure 5. Plot of Monte Carlo simulations of flux difference versus number of transfers for several CTE values.

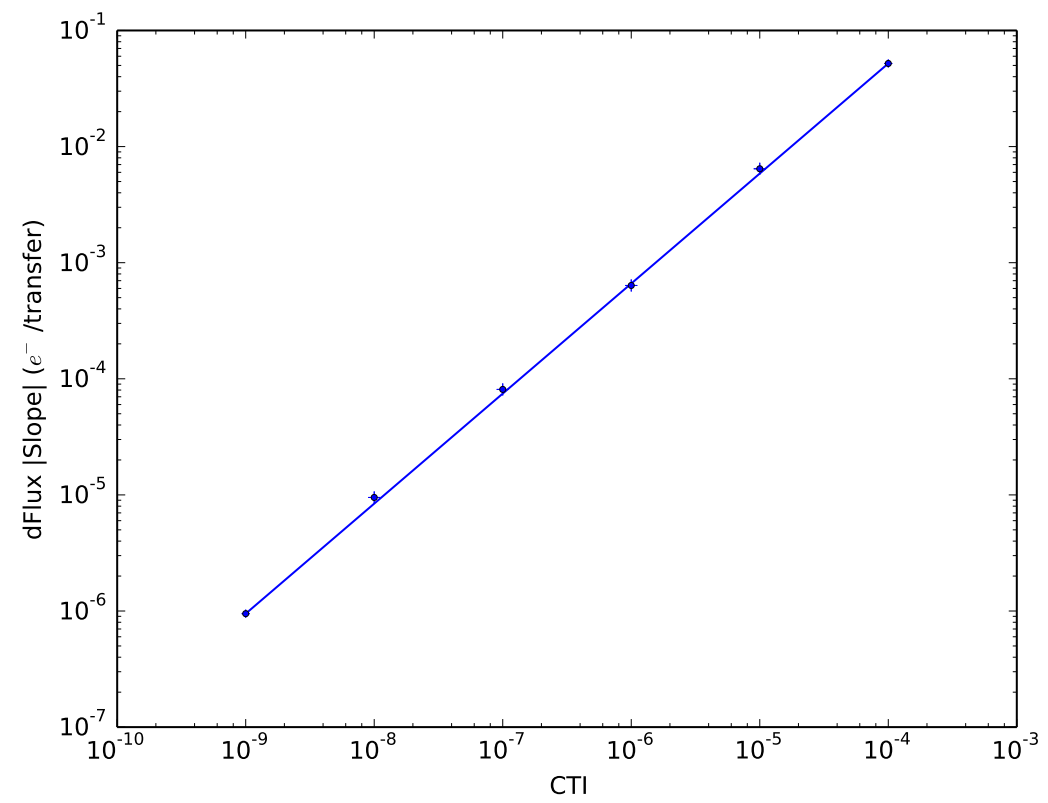

Figure 6. Plot of slope for top/bottom pixel flux differential (dFlux) versus CTI in Monte Carlo simulations, see the text. 
Similar studies were performed for three other variables: central pixel flux and two types of integral of flux as defined above. The dependence of the variables on the number of transfers was approximated with a linear function for different CTE values and the corresponding slopes were determined. The dependence of CTI on the slope for these three variables are shown in figure 7 together with the previous result on the differential flux. The central pixel and integral flux variables also show good, close to linear correlation with CTI and, hence, a good potential as possible estimators for the CTE measurement. These graphs can be used to translate the measurements of the above observables to corresponding CTE values. The other estimator of the flux integral was calculated using ngmix as described above and is denoted as the ngmix data set in figure 7. Since the fitting was involved, the results were not very stable for the smallest values of CTI so only 4 points are presented for this variable. However it shows that there exists good correlation between the slope versus associated CTI for higher CTI values, as with the other possible estimators tested here.

One can conclude from figure 7 that the differential flux and central pixel flux are similar in performance and both result in larger number of electrons per transfer for a given CTI compared to the integral flux. We leave for the future their detailed comparison in terms of sensitivity to CTI, which should also take into account uncertainties of the estimators to evaluate the corresponding signal-to-noise ratios.

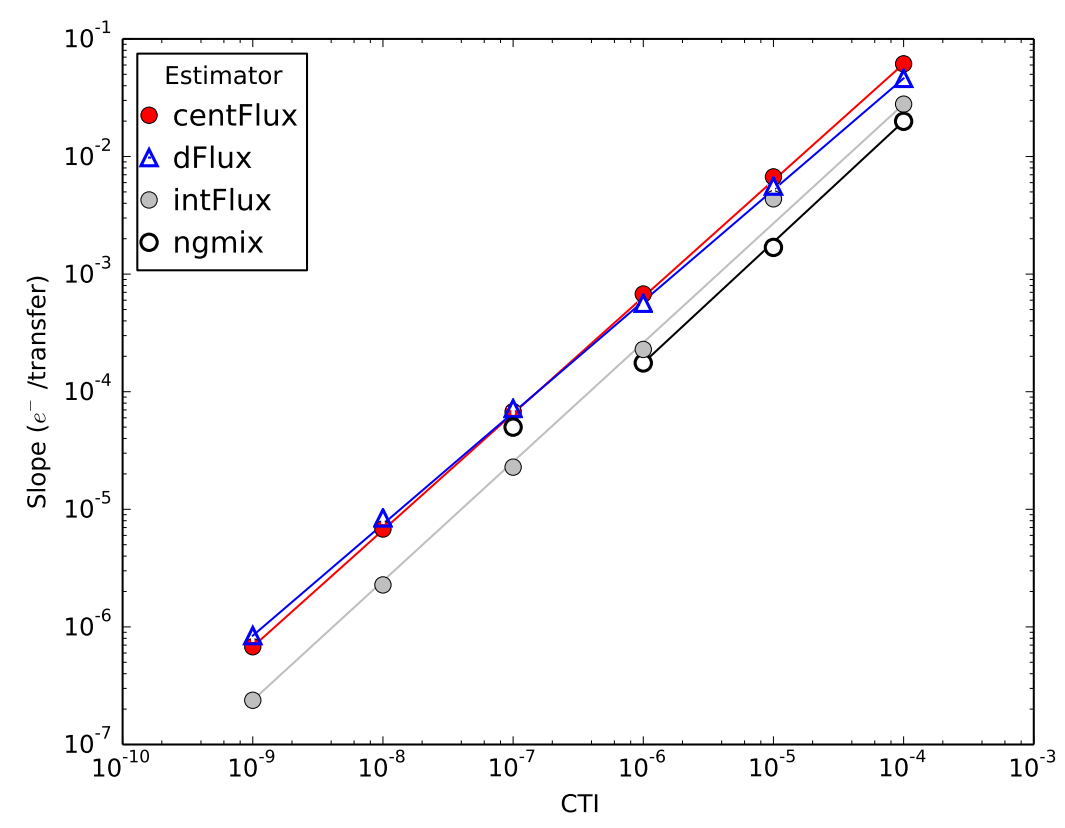

Figure 7. CTI as function of three observables based of differential flux, central pixel flux, integral flux, and flux amplitude from 2-D Gauss fit in Monte Carlo simulations, see the text.

It was determined through similar studies that the hit ellipticity did not show a consistent relationship between the CTI and varying number of transfers, especially for CTI value smaller than $10^{-4}$. Similarly to the ngmix integrated flux, the ellipticity measurements are extracted from the 2-D Gaussian fit on the x-ray hit shape. At higher CTE (small CTI) values, ellipticity measurements are not reliable to the required precision and become less correlated to the number of transfers. 
It should be noted that this study addresses the question if the total flux, evaluated as an integral over multiple pixels, can be used as a CTE estimator. For thick CCDs where the diffusion is considerable, the total x-ray energy deposition must be determined by integrating multiple pixels, which, in principle, could mask the CTE effects. As was shown above for the charge integration performed over $3 \times 3$ pixel area around pixel with maximum charge, the charge transfer effects still can be measured reliably though the slope of the dependence cannot be interpreted directly as CTI.

\section{CTE measurement in the ${ }^{55} \mathrm{Fe}$ data}

The same data sample of ${ }^{55} \mathrm{Fe}$ x-rays as described in section 2 was analyzed using a variation of the total hit flux analysis method. The hit finding used the optimized parameters, at least one pixel with signal exceeding noise at least by a factor of 40 and the growth factor of two, as described in section 2 . $\mathrm{X}$-ray hits were identified and then fit with a 2-D Gaussian following the procedure described in detail in [8] and [9]. Centroid coordinates of the Gaussian and total flux were recorded and hits were sorted into eleven bins of 182 pixels each in parallel (column) direction for each of 16 sensor section. The flux in each bin had a prominent $\mathrm{K}_{\alpha}$ peak, which was fit with a Gaussian as shown in figure 8 for the first bin (least number of transfers) of section \#11. The flux is measured in ADU (ADC units), which can be converted to the number of electrons using gain calibration, 3.4 electrons/ADU.

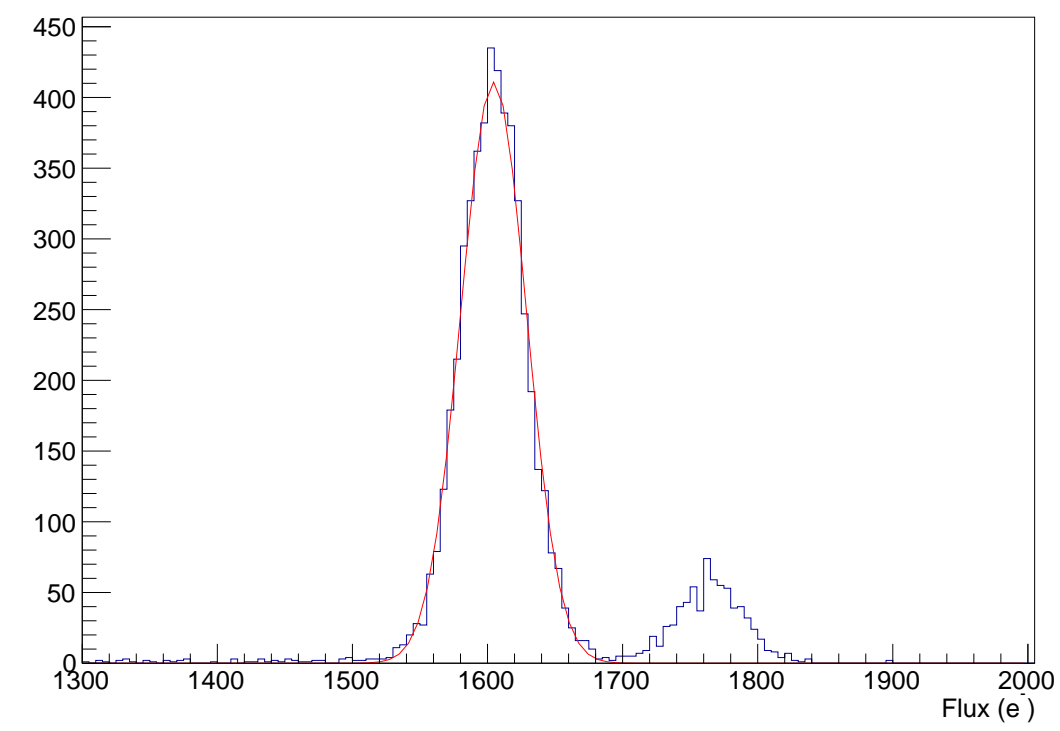

Figure 8. Flux distribution in the ${ }^{55} \mathrm{Fe}$ data in the first bin (least number of transfers) of the sensor section \#11.

The mean value of the Gaussian is an estimate of the x-ray energy deposition modified by the charge transfer effects. Its dependence on the bin number, hence on the distance along the column, is shown in figure 9 for one of 16 sensor sections (\#11). The slope of linear fit to the points expressed in electrons per transfer can be interpreted as charge lost per transfer between neighboring pixels. Note that this number cannot be interpreted as CTI (after corresponding normalization) as recommended by Janesick [3] because it does not take in to account the charge smearing due to the 
diffusion. Instead the results from the previous section in figure 7 were used to translate the lost charge to the corresponding CTI value.

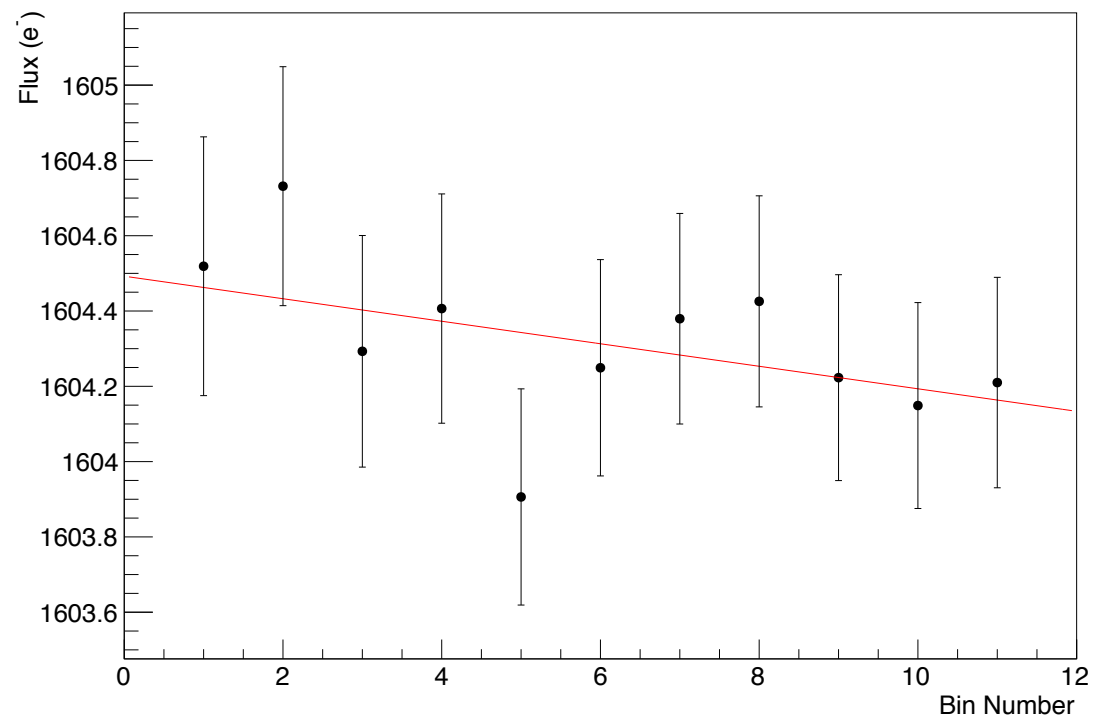

Figure 9. Mean flux values for the ${ }^{55} \mathrm{Fe}$ data for the eleven bins along the column direction for the sensor section \#11.

Figure 10 shows results of the CTE determination using the method described above for all 16 sections of the tested sensor. This measurement demonstrated that determination of the energy

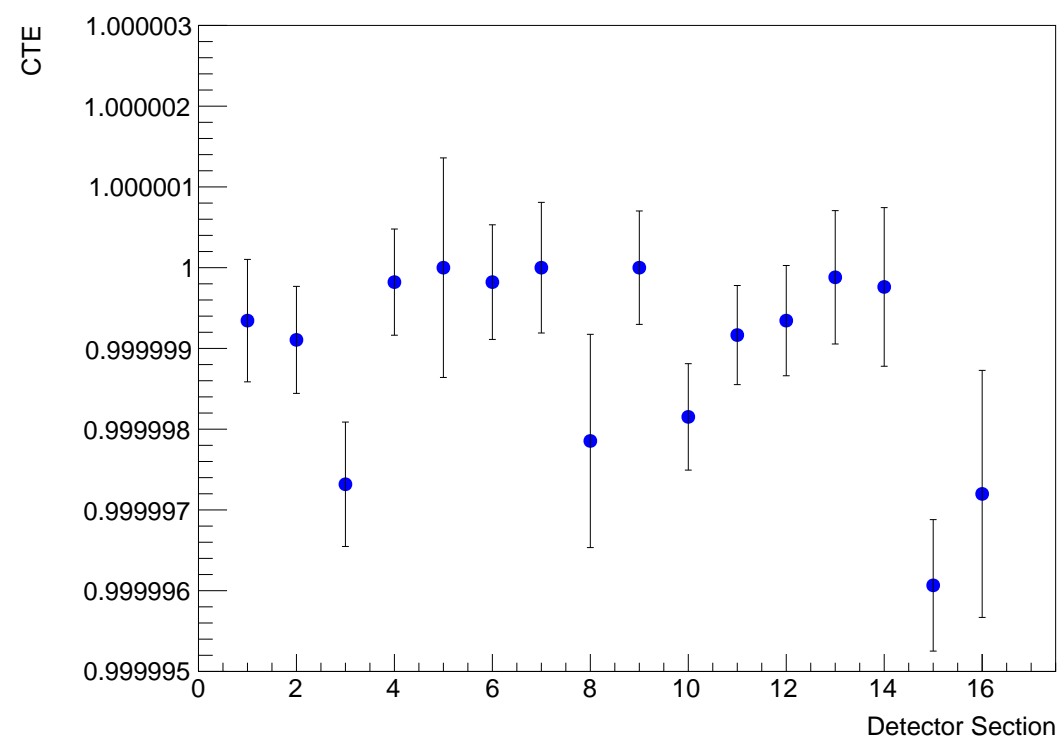

Figure 10. CTE measurements for 16 sections of the e2v-113-03 sensor using 11 bin flux analysis of ${ }^{55} \mathrm{Fe}$ data. 
deposition for the $K_{\alpha}$ peak of ${ }^{55} \mathrm{Fe}$ remains one of the most straightforward ways to measure CTE for thick, fully depleted CCDs if one takes in to account the additional charge smearing due to the diffusion. The CTE of this prototype satisfy the LSST specification for the parallel charge transfer efficiency.

\section{Conclusions}

Measurements of ${ }^{55} \mathrm{Fe}$ x-rays hits in CCDs show potential for for new variables used for determination of charge transfer efficiency: difference in flux between trailing and leading pixel, central pixel flux, integral of flux over the $3 \times 3$ pixel region and another estimate of the integral performed employing a 2D Gaussian fit. All three variables show strong correlation with CTI as was determined through Monte Carlo simulations. Hit ellipticity measurements, which require fitting the pixel values, appear to be unreliable for small CTI values.

\section{Acknowledgments}

The authors would like to thank Paul O'Connor and Merlin Fisher-Levine for their contributions to this work. This project was supported in part by the U.S. Department of Energy, Office of Science, Office of Workforce Development for Teachers and Scientists (WDTS) under the Science Undergraduate Laboratory Internships Program (SULI).

\section{References}

[1] S.M. Kahn et al., Design and development of the 3.2 gigapixel camera for the Large Synoptic Survey Telescope, Proc. SPIE 7735 (2010) $77350 \mathrm{~J}$.

[2] LSST Science, LSST Project collaborations, P.A. Abell et al., LSST Science Book, Version 2.0, arXiv: 0912.0201.

[3] J.R. Janesick, Scientific Charge-coupled Devices, SPIE Press, Bellingham, Washington, U.S.A. (2001).

[4] P. O'Connor et al., Integrated system tests of the LSST raft tower modules, Proc. SPIE 9915 (2016) 99150X.

[5] P.R. Jorden, D. Jordan, P.A. Jerram, J. Pratlong and I. Swindells, e2v new CCD and CMOS technology developments for astronomical sensors, Proc. SPIE 9154 (2014) 91540M.

[6] P. Kubanek et al., RTS-2 — remote telescope system, 2nd Version, AIP Conf. Proc. 727 (2004) 753.

[7] P. O'Connor et al., Characterization of prototype LSST CCDs, Proc. SPIE 7021 (2008) 702106.

[8] E. Sheldon, An implementation of Bayesian lensing shear measurement, Mon. Not. R. Astron. Soc. Lett. 444 (2014) L25.

[9] A. Nomerotski, New characterization techniques for LSST sensors, 2015 JINST 10 C06010. 